\title{
A cognitively enhanced online Tai Ji Quan training intervention for community-dwelling older adults with mild cognitive impairment: A feasibility trial
}

\author{
Fuzhong $\mathrm{Li}^{1 *}$, Peter Harmer ${ }^{2}$, Kathleen Fitzgerald ${ }^{3}$ and Kerri Winters-Stone ${ }^{4}$
}

\begin{abstract}
Background: This study examines the feasibility, acceptability, and safety of a newly developed cognitive-enhancing Tai Ji Quan training intervention, delivered via remote videoconferencing, for older adults with mild cognitive impairment (MCl).

Methods: In a three-arm feasibility trial, community-dwelling older adults with $\mathrm{MCl}(\mathrm{N}=69$; mean age $=74.6$ years, $57 \%$ women) were randomized to a cognitively enhanced Tai Ji Quan $(n=23)$, standard Tai Ji Quan $(n=22)$, or stretching group $(n=24)$ and participated in a 60-minute online exercise session via Zoom, twice weekly for 16 weeks. Participants were recruited primarily in the state of Oregon through mass mailing and word of mouth. The primary outcomes were intervention feasibility (with respect to recruitment, online intervention delivery, fidelity and compliance, and attrition and retention rates), acceptability, and safety. We also assessed feasibility of online data collection and test-retest reliability and explored preliminary trends on secondary outcomes that included global cognitive function, dual-task cost, and domain-specific cognition function.
\end{abstract}

Results: The study had an average recruitment rate of 55\%. Feasibility was demonstrated by the overall successful online program implementation, with good fidelity, acceptable compliance (76\%), and excellent retention (94\%). The cognitively enhanced Tai Ji Quan intervention was shown to be acceptable to participants as well as safe, with no major intervention-related moderate/severe events. At week 16, the group receiving cognitively enhanced Tai Ji Quan training showed a positive trend in the cognitive function and dual-task outcome measures whereas the group receiving standard Tai Ji Quan training exhibited positive trends on global and domain-specific cognitive measures.

Conclusions: Preliminary findings of this pilot study indicate the feasibility, acceptability, and safety of a tailored, cognitively enhanced Tai Ji Quan training intervention delivered remotely to home settings via videoconferencing for community-dwelling older adults with $\mathrm{MCl}$.

Trial registration: Clinicaltrials.gov identifier NCT04070703

Keywords: Cognitive impairment, Dual-task, Elderly, Exercise, Tai Chi, Telehealth

*Correspondence: fuzhongl@ori.org

1 Oregon Research Institute, Eugene, Oregon, OR 97403, USA

Full list of author information is available at the end of the article

\begin{abstract}
Background
Age-related decline in cognitive function has long been recognized as an important part of the aging process [1]. Early symptoms of cognitive decline include memory loss [2] that may lead to a condition commonly known as
\end{abstract}

(c) The Author(s) 2022. Open Access This article is licensed under a Creative Commons Attribution 4.0 International License, which permits use, sharing, adaptation, distribution and reproduction in any medium or format, as long as you give appropriate credit to the original author(s) and the source, provide a link to the Creative Commons licence, and indicate if changes were made. The images or other third party material in this article are included in the article's Creative Commons licence, unless indicated otherwise in a credit line to the material. If material is not included in the article's Creative Commons licence and your intended use is not permitted by statutory regulation or exceeds the permitted use, you will need to obtain permission directly from the copyright holder. To view a copy of this licence, visit http://creativecommons.org/licenses/by/4.0/. The Creative Commons Public Domain Dedication waiver (http://creativeco $\mathrm{mmons}$.org/publicdomain/zero/1.0/) applies to the data made available in this article, unless otherwise stated in a credit line to the data. 
mild cognitive impairment (MCI) [3]. Progressive deterioration of MCI will ultimately put aging adults, whose health and quality of life are often compromised by acute or chronic health conditions, at high risk for developing or progressing to Alzheimer's disease or a related dementia $[3,4]$. Successfully addressing this global public health crisis $[5,6]$, however, requires intervention strategies that can effectively prevent or delay any decline in cognitive function, thus decreasing dementia risk [7].

Non-pharmacological interventions, such as physical exercise, have been shown to improve cognitive performance, including memory, processing speed, and executive function in older adults with and without cognitive impairment [8-10]. Research suggests that the cognitive benefits may be further enhanced through integration of concurrent cognitive and motor task activities, that is, dual-task training [11-13]. In this respect, Tai Ji Quan, which requires a significant amount of cognitive effort allocated to the performance and mastery of intricate whole-body movements $[14,15]$, appears to fit well as a cognitive and motor training therapy for preventing or mitigating memory loss and cognitive decline among older adults with MCI [16-18].

Contemporary Tai Ji Quan training approaches, which primarily emphasize training participants to master Tai Ji Quan forms and movements [14], do not explicitly integrate the cognitively demanding features of this mindbody therapy into a multitasking exercise that could be cognitively stimulating and mentally beneficial for the MCI population, which experiences increasing difficulties in performing dual-task functions in activities of daily living $[19,20]$. In addition, existing Tai Ji Quan intervention delivery methods are primarily group-based with in-person administration. This approach can create barriers for practical implementation and broad dissemination, especially in extenuating circumstances, such as the COVID-19 pandemic, which has significantly restricted daily life activities of older adults, including attending community-based exercise classes [21].

In an effort to integrate cognitive activities explicitly into Tai Ji Quan training in order to maximize its cognitive training benefits, we developed a cognitively enhanced Tai Ji Quan intervention, delivered online via Zoom videoconferencing, for community-dwelling older adults with MCI. In this pilot study, our objectives were to (a) examine the feasibility (with respect to recruitment, online intervention delivery, fidelity, compliance, and attrition and retention rates), acceptability, and intervention safety of the newly developed intervention, (b) assess the feasibility of online data collection and test-retest reliability, and (c) explore preliminary trends in cognitive function and dual-task cost data to inform future full-scale trials.

\section{Methods}

\section{Study Design}

We performed a single-blind, 3-arm, parallel design, randomized feasibility trial with a 1:1:1 allocation ratio. The feasibility study was conducted in conjunction with an on-going trial (Clinicaltrials.gov identifier NCT04070703; 28/08/2019), with the research protocol approved by the institutional review board of the Oregon Research Institute. The study was performed between August 2020 and September 2021, during the 2020-2021 phase of the COVID-19 pandemic, with all research activities (recruitment, enrollment, intervention, and data collection/assessment) performed remotely via the use of telephones (for prescreening) and online conferencing software (Zoom.com) (for trial implementation and outcome assessment). All participants provided informed consent, completed electronically via online Qualtrics survey software (qualtrics.com). The feasibilitytrial reporting follows the general guidelines described in the Consolidated Standards of Reporting Trials (CONSORT) 2010 statement: Extension to randomized pilot and feasibility trials [22].

\section{Setting, Participants, Recruitment, and Procedures}

The study was undertaken in home settings where the target population was community-dwelling, independent-living older adults. Initial enrollment efforts targeted individuals who lived in Oregon but was expanded to other states in the U.S. after the first wave of recruitment. Multiple methods of recruitment were used, including direct mailing, online promotions (e.g., research website, social media), word of mouth, and contact with volunteers from previous research projects.

The recruitment criterion was community-dwelling adults aged 65 years or older who met diagnostic criteria for having MCI [23]. Evidence of MCI was established if individuals (a) self-reported a change or decline in memory (corroborated by an informant), (b) recorded a score of $\leq 0.5$ on the Clinical Dementia Rating (CDR) [24] scale (with information derived from both the participant and an informant), and (c) showed no major impairment in cognitive function as indexed by a score of $\geq 24$ on the Mini Mental State Evaluation (MMSE) [25]. We excluded individuals who (a) had participated in a rigorous and structured physical activity or exercise program (including Tai Ji Quan) in the past 3 months, (b) showed signs of depression as indicated by a score of $\geq 5$ on the Geriatric Depression Scale [26], (c) had major medical or physical conditions that precluded exercise, as determined by their healthcare practitioner, or (d) were unable to follow the consent process or sign the study consent form.

Recruitment was conducted primarily via initial phone communication, followed by a scheduled online 
Zoom meeting using a procedure established in a prior study [27]. Briefly, a research assistant made initial contact via a telephone call with those who responded to the study promotion. This contact confirmed eligibility criteria regarding age, memory status, levels of habitual physical activity, and time availability. An independent interview was conducted with an informant who provided information regarding the respondent's cognitive status based in part on a CDR assessment [24]. Individuals were further evaluated, via an online Zoom session, on additional eligibility criteria, including administration of the CDR and MMSE. During each stage of prescreening, individuals were given sufficient time to ask questions and clarify issues related to risk, benefits, and commitment related to study participation. Those who met the study eligibility criteria signed the study consent form, and then underwent an online baseline assessment session conducted by an independent assessor. The recruitment for the feasibility study ended, as planned, in April 2021.

\section{Randomization and Blinding}

Eligible individuals were randomly assigned in a 1:1:1 ratio to receive one of the three active interventions, with a block size of 4 or 6 . Randomization was not stratified. The randomization list was generated by a research data analyst with the use of nQuery software (www.statsols. com) and was kept in sealed envelopes. Allocation concealment was ensured since the randomization was performed by a research assistant who did not know the sequence of interventions and was not involved in the assessment or intervention. Randomization occurred after informed consent was obtained and baseline assessments were completed.

Participants were not blinded to the intervention group allocation but were requested not to disclose their group allocation to the study assessors during all follow-up assessments. Outcome assessors were masked to group allocation throughout the study period. Blinding was strictly maintained by (a) emphasizing to assessors the importance of minimizing assessment bias, (b) regular checking of the blinding status via inquiries made to randomly selected participants, and (c) randomly asking assessors to guess what intervention the participants had received. Every effort was made to keep the assessors from knowing the group allocation, including the use of identical assessment forms, assigned study participation identification numbers, and a designated database separate from the one used by unblinded study recruiters. In instances when blinding was broken, the assessor was immediately excused and replaced by another blind assessor.

\section{Intervention}

\section{Cognitively Enhanced Tai Ji Quan Training}

The intervention was adapted from the Tai Ji Quan: Moving for Better Balance program [14, 28], augmented by a set of cognitively stimulating exercises practiced concomitantly with Tai Ji Quan to increase cognitive processing demands [27, 29]. The progressive training consisted of 5-15 minutes of preparatory (warm-up) exercises, 45-55 minutes of core training (learning, practicing, repeating) in the 8-form routine [28], and 1-2 minutes of cool-down exercise. During the initial 10 weeks, the core physical exercise involved primarily learning and repeatedly practicing the 8 forms (in a prespecified sequence of Moving a Ball, Part Wild Horse's Mane, Repulse Monkey, Brush Knees, Fair Lady Works the Shuttle, Grasp Peacock's Tail, Waving Hands like Clouds, and Single Whip), with training focusing on symmetrical and coordinated actions such as trunk rotation and weight shifting, controlled and coordinated displacement of the body's center of mass over the base of support, dynamic eyes-head movements, and anterior-posterior and medial-lateral stepping. From week 10 on, training became fully integrated with prespecified cognitive exercises, described below, that targeted domains of memory, executive function, and processing speed.

The training approach used a mix of dual-task, task prioritization, and memory exercises intended to actively engage participants in learning and practicing Tai Ji Quan forms and variations of forms and encouraging instructor-participant interaction during practice. Specifics of integrated Tai Ji Quan exercises included (a) verbalizing step-by-step movements involved in a form, (b) responding to correct form instructions while inhibiting unwanted instructions, (c) exercising form recalls (including 2 or 3 forms back) either standing (with a narrow base of support) or moving, (d) practicing a sequence of forms while verbally associating form names with form numbers (or the reverse), (e) performing forms without verbal or visual instructional cues, and (f) performing word spelling (forward and backward), side-switching (alternating between lateral and bilateral sides), out-ofsequence form switching, and varying form sequences (forward, backward, oddly or evenly ordered).

\section{Standard Tai Ji Quan Training}

This intervention consisted of the identical 8-form routine training protocol [28] implemented for the cognitively enhanced Tai Ji Quan group, except that the cognitive exercises were not included. Instead, an integrated set of therapeutic movement exercises was added that focused on training of sensory integration, mobility, and limits of stability and recovery of near loss of 
balance. These exercises followed the general guidelines regarding balance training for older adults, with activities that emphasized progressively difficult postures with varying sizes of the base of support, postural perturbation that displaced the body away from equilibrium, weightloading on lower-extremity postural muscle groups, and sensory compensation and integration [30].

The intervention followed a traditional Tai Ji Quan training approach, where instructors play a dominant and leading role in teaching and practicing, without instructor-participant interaction during exercise. Accordingly, each exercise session primarily involved teaching participants, via both verbal and visual cues, Tai Ji Quan forms and movements through repeated practice. The specifics of physical training activities included controlled, selfinitiated Tai Ji Quan-based exercises with synchronized breathing, including center-of-gravity displacement using a dynamic interplay of stabilizing and self-induced destabilizing postural actions involving unilateral weightbearing and weight-shifting movements, trunk and pelvic rotation, ankle sway, multidirectional stepping, and eyehead-hand movements [28]. Similar to the protocol of the cognitively enhanced group, the exercise training in this intervention was taught progressively, with learning and practice of the 8 individual forms taking place during the first 10 weeks and repeated practice with variations thereafter.

\section{Stretching Exercise}

This intervention, with the protocol adopted from our prior trial [28] and piloted in another study [27], consisted of various muscle stretches, performed in either seated or standing positions. The teaching strategy was identical to that of standard Tai Ji Quan training in that participants were asked to follow instructors' instructions in performing all exercise activities specified in the program, and no instructor-participant interaction was encouraged. Each session began with light warmup exercises involving arm, neck, and leg circles; trunk rotation; and light walking. The core part of the exercises involved stretches of the upper body (neck, arms, back, shoulders, and chest) and lower extremities (quadriceps, hamstrings, calves, and hips), along with slow and gentle trunk rotations. Each exercise session ended with breathing exercises and progressive relaxation of major muscle groups.

\section{Online Intervention Delivery Protocol}

The three active exercise interventions were delivered using Zoom, for 16 weeks, with the same length of time (60 minutes per class session) and frequency (twice weekly). On each class day, participants were given a secured Zoom link via e-mail that allowed them to access the scheduled online exercise session. Participants were encouraged to sign into the online class 15 minutes before it started and, as a human subjects protection feature, they were admitted to the class by either the class instructor or project staff. After signing in, participants were instructed to (a) turn on video and (b) use the Speaker View feature in Zoom for better viewability of exercise instruction. During the class, participants in the standard Tai Ji Quan and stretching classes were encouraged to mute their audio for better audio quality. This was not done for those in the cognitively enhanced Tai Ji Quan group because of the need for interaction between the instructor and participants.

Exercise instructors trained by the first author taught all exercise sessions. All instructors were recruited from the community and had at least 10 years of teaching exercise or Tai Ji Quan experience. For consistency, in all three interventions, each session consisted of warm-up exercises, core exercises, and a brief cool-down activity. Exercise intensity in each intervention group was monitored through a subjective measure of perceived exertion, ranging from light to moderate for both Tai Ji Quan groups and light for the stretching group.

We began the study with a maximum of 8 participants per class to optimize viewability in the Zoom environment. We gradually increased the maximum number of participants in a class to $12-15$ in subsequent waves of classes. We set the instructor's teaching space to an area approximately 4 feet by 4 feet square in order to provide (a) onscreen viewability, (b) instructional clarity, and (c) effective space utilization and movement safety. On the participant end, we set an optimum physical distance of 8-10 feet for personal computer users and 6-8 feet for iPad or smart phone users for better viewability. Participants in each group were asked to have an armless chair set aside for use during exercise.

\section{Measurements \\ Primary Outcomes}

Intervention fidelity. The intervention fidelity, as part of the quality control over intervention delivery, was defined as the extent to which interventionists successfully implemented the prespecified teaching protocol [31]. Our fidelity evaluation included (a) successful completion of the 60-minute session delivered twice weekly over a 16-week period, (b) the instructor's adherence to the teaching and training protocol described in detail in our predeveloped teaching manual, and (c) an overall class attendance rate of $\geq 75 \%$ (out of the total planned 32 intervention class sessions). The items in a and b were monitored weekly by means of observing class attendance and conducting a weekly online class fidelity check. Items in $\mathrm{a}$ and $\mathrm{b}$ were also monitored by means of class 
recordings, which included an evaluation of the instructor's faithfulness in implementing the key components of the interventions, involving warm-up, core exercise training, cool-down, and clarity in verbal and visual instructions. The fidelity check outcomes were as follows: (a) Pass Satisfactorily (faithful implementation), (b) Needs Attention (signs of deviations or drift and, therefore, adjustments needed), or (c) No Pass (in-service training needed) (see below).

Monthly in-service training sessions conducted by the first author were planned to assure the quality of delivery. The training involved reviewing sessions taught in the previous weeks, correcting observed interventionist drift from the prespecified teaching activities/forms, reinforcing appropriate teaching techniques and steps (i.e., demonstration, learning/practice, repetitions, emphasis of key training points), doses (exercise sets, repetitions), and quality (visual and verbal instructions and presentation). The sessions also included a brief discussion of any safety issues related to the teaching and practicing of movements in the teaching manual.

Intervention attrition and study retention. On the basis of our prior trials [27, 28, 32], we set a satisfactory a priori intervention attrition rate (i.e., participants who dropped out of the assigned intervention class before the 16 -week termination) at $\leq 15 \%$. A drop from the exercise class (intervention dropout) was defined as declining to continue participating in the class exercises either by notifying project staff (via e-mail or telephone) or not responding to any class participation reminders sent by project staff. We aimed at a $90 \%$ rate of retaining and assessing randomized participants with valid outcome data (i.e., with an expected $\leq 10 \%$ missing or incomplete on cognitive and dual-task gait performance outcomes) during the 16-week study period. The same definition for intervention dropout was applied to determine the study dropout rate.

Acceptability. To assess the exercise program acceptability for the newly developed cognitively enhanced online Tai Ji Quan intervention, participants assigned to this group were asked to complete a 5-item attitudinal scale upon completing the class. The survey asked participants to rate their opinions about the class in the areas of exercise safety, challenge/enjoyment, appropriateness of exercise intensity, helpfulness in improving brain health, and overall program satisfaction. For the acceptability evaluation by the participants assigned to our cognitively enhanced Tai Ji Quan intervention group, we specified an a priori overall satisfaction level of $\geq 70 \%$.

Adverse events monitoring. Throughout the study period, both intervention- and non-intervention-related adverse events were closely monitored and recorded by research staff and adjudicated by the principal investigator of the study. We classified adverse events in three categories: Mild (i.e., events that required no medical treatment or were non-life threatening), Moderate (i.e., events that required medical treatment but were not immediate life-threatening conditions, and Serious (i.e., events that resulted in death or were life threatening and required medical treatment, including hospitalization, or significant disability/incapacity). For all events observed or reported, we further classified them into three categories in relation to the intervention: Unrelated (an event that was reported but not directly related to participation in the intervention), Possible (an event that was observed during a class that was considered likely to be associated with participation), or Definite (an event that was observed or reported during a class and was considered directly related to participation).

\section{Data Collection of Secondary Study Outcomes}

We included a set of cognitive outcome measures ranging from global cognitive function and dual-task gait performance to several domain-specific cognitive measures. All outcome measures, which are described below, were collected remotely via Zoom.

The Montreal Cognitive Assessment (MoCA) [33] measures global cognitive function that encompasses multiple domains (attention, executive functions, memory, language, conceptual thinking, calculations, visuospatial abilities, and orientation). MoCA scores range from 0 to 30, with higher scores representing better cognitive functioning. The final MoCA scores were adjusted for level of education (i.e., 1 point was added to the total score of individuals with $\leq 12$ years of education) [33].

The Timed Up and Go (TUG) test [34] was used to assess gait under single-task and dual-task conditions [35]. During the single-task condition, participants completed a 20-foot walk that involved (a) standing up from a chair, (b) walking (10 feet forward) at normal pace to a line on the floor, (c) turning, (d) walking back (10 feet) to the chair, and (e) sitting down. The 20-foot walk was then repeated under a dual-task condition where participants were asked to walk while performing an arithmetic task (i.e., starting at the number 81 and sequentially subtracting 5 from the resulting number). No specific verbal instructions were given for prioritization of one of the walking tasks during the dual-task walking trial. Using the average score (in seconds) of each walk, a dual-task cost measure was estimated through the following methods [35]:

Dual-task cost $_{\text {baseline }}=($ dual-task walking - single-task walking) / single-task walking $\mathrm{x} 100$

Dual-task cost $_{16 \text { _weeks }}=$ (dual-task walking - singletask walking) / single-task walking $\mathrm{x} 100$ 
Change in dual-task cost $=$ dual-task cost $_{16 \text { _weeks }}-$ dual-task cost baseline $_{\text {en }}$

Change (measured in percentage) in dual-task cost from baseline was used to define dual-task cost at week 16 , with positive values indicating deteriorated dual-task performance (i.e., dual-task cost), and negative values representing an improvement in dual-task performance with respect to single-task (i.e., dual-task benefit) [35].

The four domain-specific cognitive function measures were Trail Making Test B (TMT-B) [36] (in seconds), Category Fluency for Animals [37] (executive function), Forward Digit Span [38] (attention), and Backward Digit Span [38] (working memory). In TMT-B, participants were asked to connect numbers and letters in an alternating progressive sequence (i.e., 1-A-2-B-3-C, and so on) and scoring was expressed in terms of the time (in seconds) to completion. For Category Fluency, participants were asked to generate the names of as many animals as possible in 60 seconds. In assessing Forward and Backward Digit Span, participants were verbally presented with a series of digits (e.g., 6, 2, 9, 7) at a rate of one digit per second and were asked to repeat them verbatim. If participants succeeded, they were given a longer list (e.g., 5, 3, 8, 1, 6). The number of digits increased by one until the participant consecutively failed two trials of the same digit span length. In the Forward Digit-Span, participants were asked to repeat the digits in the given called order whereas in the Backward Digit-Span participants were asked to repeat the digits in reverse. The maximum number of points for each of these tests was 16 [38].

\section{Modifications and Test Consistency}

To accommodate online assessment, modifications were made in some of the cognitive measures. Specifically, for the MoCA measure [33], in the Visuospatial/Executive section, participants were asked to verbally connect each letter to the corresponding number for the Trail Making task and draw both the object (e.g., cube) and clock on a piece of paper and, once completed, show them to the assessor for evaluation. In completing the "Read list of letters" task in the Attention section, the letter-tapping task was replaced by asking participants to count the number of A's in the list. For the TMT-B measure [36], the hand-drawing task was replaced by asking participants to verbally alternate the letters and numbers out loud, and the assessor recorded the time to complete the task.

To assess test-retest reliability, we assessed the cognitive measures twice, using a 2 -week interval. Testretest reliability of the TUG measure was reported previously [27].

\section{Other Measures}

In addition, we conducted a study health survey that contained participants' demographic information, health or medical conditions, and levels of leisure physical activity [39] and depression [26]. The survey was collected via either online Qualtrics (qualtrics.com) or regular mail. Other information included self-reported weight, height, and blood pressure, which were obtained during an online assessment.

\section{Descriptive Data Analysis}

Participant characteristics at baseline were summarized by intervention group using mean, standard deviation, or percentage. Intervention adherence was calculated as the percentage of preplanned intervention sessions (32 total) attended by participants. Responses on acceptability were tabulated to describe participants' ratings of intervention acceptability in the cognitively enhanced Tai Ji Quan group. Similarly, observational or reported counts of any adverse events were tabulated by severity categories and their relationship to intervention. As a feasibility study, no inferential statistical analyses were performed [40]. As a result, we planned a sample of 60 (20 in each group) on the basis of our prior similar work [27].

For descriptive purposes, baseline and 16-week data related to cognitive and dual-task outcomes are described for each intervention group, with means and standard deviations. Trends data (i.e., differences between baseline and 16-week data) are presented as change scores, along with $95 \%$ confidence intervals. The intent-to-treat principle as used with all available data presented at baseline and 16 weeks. We performed a test-retest reliability analysis using the intraclass correlation coefficient (ICC) values. All data were processed or analyzed, where appropriate, using SPSS version 20 (SPSS, Inc.).

\section{Results}

Between August 2020 and April 2021, a total of 127 individuals were screened for eligibility; 70 were found eligible (a total recruitment rate of 55\% [70/127]) and were subsequently randomized to one of the three intervention groups (Fig. 1). As part of our prescreening protocol for memory and cognitive-related impairment, we were also able to recruit the informant for these 70 participants. One participant was removed after withdrawing consent from the study, leaving a total of 69 participants who received an intervention. Baseline characteristics of the randomized participants by intervention groups are described in Table 1.

The mean age for the study sample was 74.6 years (SD $=5.6$; range $=65-92$ years old), $57 \%$ were women, with $1.4 \%$ of participants being of Hispanic origin and $93 \%$ 


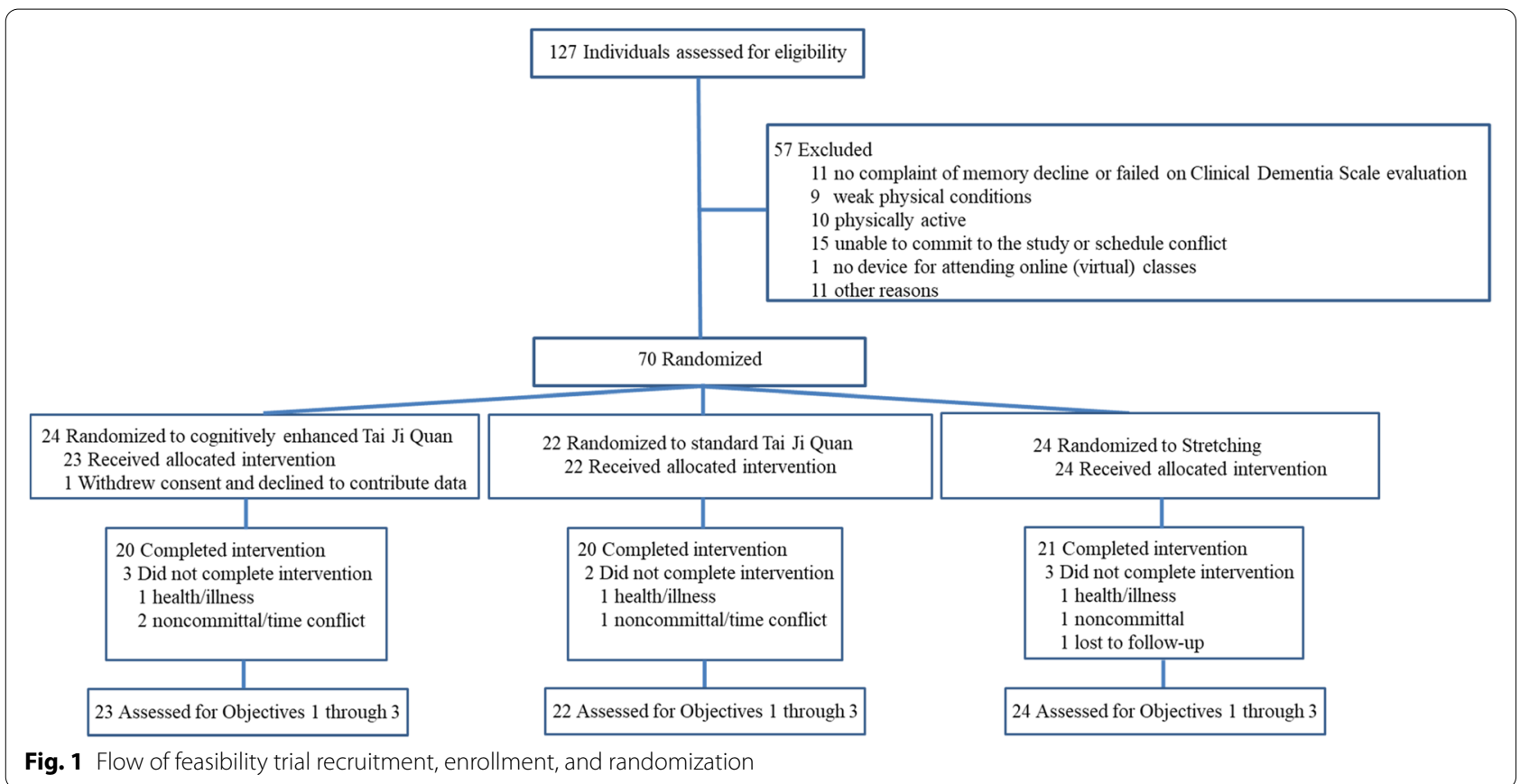

white. A total of $45(65 \%)$ of the participants reported having 2 or more chronic conditions (e.g., arthritis, heart disease, high blood pressure, osteoporosis), and 24 (35\%) participants reported taking 2 or more prescription medications. The sample had a mean (SD) MoCA score of 25.09 (2.47) and a mean (SD) MMSE score of 26.93 (1.97), with a relatively slow dual-task TUG performance (16.72 seconds [3.53]).

\section{Online Intervention Delivery}

The 16-week online delivery of all three exercise intervention classes was successful with respect to the quality of exercise instructions by intervention instructors and their ability to implement the manualized online teaching protocols in an online environment. We also did not observe major interruptions or obstacles with respect to (a) receiving Zoom links from the project, signing on to Zoom, and operating Zoom (e.g., audio, video, viewability), (b) visibility and clarity in seeing the instructor's teaching of exercise movements, or (c) availability of viewing devices for the class (i.e., personal computer, iPad, or Smart phones). While all study participants had Internet access and were able to attend classes, issues related to Internet connectivity occurred irregularly, a situation mostly impacted by either speed or weather conditions. Only one participant borrowed an iPad from us for class use. Because of the online delivery, the protocol allowed participation by those $(\mathrm{n}=7)$ who were traveling or were away from their main homes during the course of the study.

\section{Intervention Fidelity and Compliance}

Intervention fidelity was demonstrated by the fact that (a) all instructors successfully delivered the twice weekly, 16-week interventions and (b) there was an average compliance (class participation) rate of $\geq 75 \%$. The average intervention attendance rate across the 16-week period for all intervention participants was $75.8 \%$ (76.6\% in the cognitively enhanced Tai Ji Quan group, $75.5 \%$ in the standard Tai Ji Quan group, and $75.3 \%$ in the stretching exercise group). This rate met our a priori expectations. In addition, our weekly fidelity check on implementing intervention activities showed that all instructors passed the fidelity check satisfactorily and were able to follow and deliver the assigned intervention consistently and reliably per the activities detailed in the teaching plan.

\section{Intervention Attrition and Study Retention}

A total of 8 participants dropped out of the exercise intervention, resulting in an overall attrition rate of $11 \%$ (4\% less than anticipated). Reasons for withdrawing were health-related $(\mathrm{n}=3)$, noncommittal (including time conflict) $(\mathrm{n}=4)$, and lost to follow-up $(\mathrm{n}=$ 1) (Fig. 1). On the study outcome data ascertainment, 65 participants $(94 \%)$ provided both baseline data and end-point data at week 16 . We lost 4 participants $(6 \%)$ at the 16-week follow-up assessment due to change in health conditions $(\mathrm{n}=2)$, being unwilling to complete $(\mathrm{n}=1)$, and lost to follow-up $(\mathrm{n}=1)$. 
Table 1 Demographic characteristics of study participants by intervention group at baseline

\begin{tabular}{|c|c|c|c|}
\hline Characteristic & $\begin{array}{l}\text { Cognitively Enhanced Tai Ji Quan } \\
(\mathrm{n}=23)\end{array}$ & $\begin{array}{l}\text { Standard Tai Ji Quan } \\
(\mathrm{n}=22)\end{array}$ & $\begin{array}{l}\text { Stretching } \\
(\mathrm{n}=24)\end{array}$ \\
\hline Age - yr., mean (SD) & $74.4(5.1)$ & $74.5(5.6)$ & $74.9(6.3)$ \\
\hline Female sex, No. (\%) & $16(69.6)$ & $8(36.4)$ & $15(62.5)$ \\
\hline \multicolumn{4}{|l|}{ Race/Ethnicity, ${ }^{\mathrm{a}}$ No. (\%) } \\
\hline Non-Hispanic white & $22(95.7)$ & $21(95.5)$ & $21(87.5)$ \\
\hline African American & $1(4.3)$ & 0 & 0 \\
\hline Asian & 0 & 0 & $1(4.2)$ \\
\hline Hispanic & 0 & 0 & $1(4.2)$ \\
\hline Other & 0 & $1(4.5)$ & $1(4.2)$ \\
\hline \multicolumn{4}{|l|}{ Education, №. (\%) } \\
\hline High school diploma or lower & $12(52.2)$ & $10(45.5)$ & $13(54.2)$ \\
\hline College degree or higher & $11(47.8)$ & $12(54.5)$ & $11(45.8)$ \\
\hline Body mass index, mean (SD) & $29.9(6.10)$ & $27.7(5.05)$ & $27.9(5.77)$ \\
\hline \multicolumn{4}{|c|}{ Resting blood pressure - mm Hg, mean (SD) } \\
\hline Systolic & $125.65(13.71)$ & $126.73(9.09)$ & $125.92(14.33)$ \\
\hline Diastolic & $73.78(6.42)$ & $72.91(6.74)$ & $72.42(8.68)$ \\
\hline Depression, ${ }^{C}$ mean (SD) & $3.09(1.31)$ & $3.23(1.66)$ & $3.33(1.24)$ \\
\hline MMSE, mean (SD) & $26.96(1.87)$ & $26.95(1.91)$ & $26.88(2.19)$ \\
\hline \multicolumn{4}{|c|}{ Self-reported chronic conditions, No. (\%) } \\
\hline None & $2(8.7)$ & $1(4.5)$ & $1(4.2)$ \\
\hline 1 & $5(21.7)$ & $10(45.5)$ & $5(20.8)$ \\
\hline 2 & $5(21.7)$ & $4(18.2)$ & $5(20.8)$ \\
\hline$\geq 3$ & $11(47.8)$ & $7(31.8)$ & $13(54.2)$ \\
\hline \multicolumn{4}{|c|}{ Self-reported medication use, №. (\%) } \\
\hline None & $7(30.4)$ & $7(31.8)$ & $7(29.2)$ \\
\hline 1 medication & $9(39.1)$ & $7(31.8)$ & $8(33.3)$ \\
\hline 2 medications & $5(21.7)$ & $6(27.3)$ & $4(16.7)$ \\
\hline$\geq 3$ medications & $2(8.8)$ & $2(9.1)$ & $5(20.8)$ \\
\hline
\end{tabular}

a Participants could respond "Yes" to more than one race/ethnic group.

${ }^{b}$ Calculated as the weight in kilograms divided by the square of the height in meters. SD: standard deviation.

' Geriatric Depression Scale (short form) consisting of 15 items (range: 0 to 15); 0-4 is considered normal, depending on age, education, and complaints; 5-8 indicates mild depression; 9-11 indicates moderate depression; and 12-15 indicates severe depression

\section{Intervention Acceptability}

For the cognitively enhanced online Tai Ji Quan group, descriptive statistics showed that $77 \%$ of the participants "strongly agreed" ("5") and an additional 23\% "agreed" ("4") that the exercise program was safe to practice. A total of 55\% "strongly agreed" and $45 \%$ "agreed" that the exercises were challenging but enjoyable. A total of 59\% "strongly agreed" and 36\% "agreed" that the intensity of the exercise program was appropriate and manageable. A total of $64 \%$ rated the program as "extremely helpful" for brain health, with $27 \%$ finding it "very helpful" and 9\% finding it "moderately helpful." Finally, on overall satisfaction, $82 \%$ were "extremely satisfied" and $18 \%$ were "somewhat satisfied" with the program (Table 2).

\section{Adverse Events}

No serious adverse events were documented over the 16 -week study period (Table 3). A total of five moderate events (related to medical surgeries or conditions) were reported, none of which was intervention related. Of the six mild events reported, four were either possibly or definitely intervention related. For example, 1 participant in the cognitively enhanced Tai Ji Quan group reported a hernia pain caused by the exercise and 3 participants complained about muscular discomfort or pain. Of the 11 participants who reported any adverse events, all but 3 successfully completed the assigned intervention and outcome assessment. 
Table 2 Intervention acceptability rated by participants in the cognitively enhanced online Tai Ji Quan intervention ( $\left.\mathrm{N}=22^{\mathrm{a}}\right)$

\begin{tabular}{|c|c|c|c|c|c|}
\hline Item/Rating Scale & Strongly agree & Agree & Neutral & Disagree & Strongly disagree \\
\hline $\begin{array}{l}\text { 1. I found the exercise pro- } \\
\text { gram safe to practice }(n, \%)\end{array}$ & $17(77)$ & $5(23)$ & 0 & 0 & 0 \\
\hline $\begin{array}{l}\text { 2. I found the exercises chal- } \\
\text { lenging but enjoyable }(n, \%)\end{array}$ & $12(55)$ & $10(45)$ & 0 & 0 & 0 \\
\hline \multirow{2}{*}{$\begin{array}{l}\text { 3. I found the intensity of the } \\
\text { exercise program appropriate } \\
\text { and manageable }(n, \%)\end{array}$} & $13(59)$ & $8(36)$ & $1(5)$ & 0 & 0 \\
\hline & Extremely helpful & Very helpful & Moderately helpful & Little helpful & Not at all helpful \\
\hline \multirow{2}{*}{$\begin{array}{l}\text { 4. How helpful did you find } \\
\text { the exercise program to } \\
\text { improve your brain health } \\
\text { overall? (n, \%) }\end{array}$} & $14(64)$ & $6(27)$ & $2(9)$ & 0 & 0 \\
\hline & Extremely satisfied & Somewhat satisfied & $\begin{array}{l}\text { Neither satisfied nor dissatis- } \\
\text { fied }\end{array}$ & Somewhat dissatisfied & Extremely dissatisfied \\
\hline $\begin{array}{l}\text { 5. Overall, how satisfied } \\
\text { were you with the exercise } \\
\text { program? }(n, \%)\end{array}$ & $18(82)$ & $4(18)$ & 0 & 0 & 0 \\
\hline
\end{tabular}

a One participant was unable to complete the survey due to health reasons

Table 3 Number of adverse events reported during the 16-week study period by intervention group $(N=69)$

\begin{tabular}{|c|c|c|c|c|c|c|c|c|c|}
\hline \multirow{4}{*}{ Nature of event ${ }^{*}$} & \multicolumn{9}{|c|}{ Intervention Group } \\
\hline & \multicolumn{9}{|c|}{$\begin{array}{l}\text { Cognitively Enhanced } \\
\text { Tai Ji Quan }\end{array}$} \\
\hline & \multicolumn{3}{|c|}{ Tai Ji Quan } & \multicolumn{3}{|c|}{ Standard Tai Ji Quan } & \multicolumn{3}{|l|}{ Stretching } \\
\hline & & & & \multicolumn{4}{|c|}{ Relationship to Intervention } & & \\
\hline & Unrelated & Possible & Definite & Unrelated & Possible & Definite & Unrelated & Possible & Definite \\
\hline \multicolumn{10}{|l|}{ Serious } \\
\hline Number of events & 0 & 0 & 0 & 0 & 0 & 0 & 0 & 0 & 0 \\
\hline \multicolumn{10}{|l|}{ Moderate } \\
\hline Number of events & 1 & 0 & 0 & 1 & 0 & 0 & 3 & 0 & 0 \\
\hline \multicolumn{10}{|l|}{ Mild } \\
\hline Number of events & 0 & 1 & 1 & 1 & 1 & 1 & 0 & 1 & 0 \\
\hline
\end{tabular}

*Definition:

Mild: events that required no medical treatment and were non-life threatening (e.g., lower back pain, ankle/muscle soreness or pain, a fall without needing medical attention).

Moderate: events that required medical treatment but were not immediate life-threatening conditions (e.g., hand surgery or a medical procedure).

Serious: death or life-threatening events that required medical treatment, including hospitalization (e.g., open heart surgery or a major medical surgery that required hospital admission), or that resulted in significant disability/incapacity.

\section{Online Data Ascertainment}

We received no complaints about online assessment from participants. In addition, participants who completed their online baseline assessment $(\mathrm{N}=69)$ and 16 -week follow-up assessment $(n=65)$ encountered no major issues with respect to device/Zoom use, Internet connection, movement space for the dual-task walking measures, or safety concerns. The average time to complete the online assessment of the cognitive and gait performance measures was approximately 45 minutes. Ninety-one percent of the study participants submitted the study survey online. All study informants completed their CDR evaluation for their respective participant.

We found no major break in our blinding assessment protocol during the study. One assessor was exposed, by accident, to a participant's group assignment during scheduling for a follow-up assessment and was immediately replaced by another blinded assessor. 
Table 4 Intraclass correlation coefficients from test-retest (at a 2-week interval) of cognitive measures

\begin{tabular}{lc}
\hline Cognitive measures & \\
\hline & ICC \\
Montreal Cognitive Assessment & 0.87 \\
Trail-Making B & 0.91 \\
Digit Span - forward & 0.90 \\
Digit Span - backward & 0.89 \\
Verbal Fluency & 0.92
\end{tabular}

ICC Intraclass correlation coefficient

\section{Change in Cognitive and Dual-Task Outcomes}

Table 5 presents descriptive statistics related to the cognitive and dual-task cost measures at baseline and 16 weeks, and change scores from baseline. At week 16, a change from baseline, judging from the CI estimates, could be observed for all outcome measures for the cognitively enhanced Tai Ji Quan group. Similarly, with the exception of dual-task cost, a change was also noted on the outcome measures for the standard Tai Ji Quan training group. No change was evident on all the estimates for the stretching exercise group.

Table 5 Descriptive statistics (means and standard deviations) on cognitive and dual-task cost measures at baseline and 16 weeks, and mean change scores (with 95\% confidence intervals) from baseline

\begin{tabular}{|c|c|c|c|}
\hline \multirow[t]{2}{*}{ Cognitive and dual-task cost measure } & \multirow{2}{*}{$\begin{array}{l}\text { Cognitively } \\
\text { Enhanced } \\
\text { Tai Ji Quan } \\
(\mathbf{n}=23)\end{array}$} & \multicolumn{2}{|l|}{ Standard } \\
\hline & & $\begin{array}{l}\text { Tai Ji Quan } \\
(\mathbf{n}=\mathbf{2 2})\end{array}$ & $\begin{array}{l}\text { Stretching } \\
(\mathrm{n}=24)\end{array}$ \\
\hline \multicolumn{4}{|l|}{ Montreal Cognitive Assessment (points), mean (SD) } \\
\hline $\begin{array}{l}\text { Baseline } \\
16 \text { weeks } \\
\text { Change from baseline (16 weeks - baseline), (95\% Cl) }\end{array}$ & $\begin{array}{l}25.04 \pm 2.87 \\
27.39 \pm 1.37 \\
2.35(1.18 \text { to } 3.51)\end{array}$ & $\begin{array}{l}25.09 \pm 2.43 \\
26.82 \pm 1.84 \\
1.73(0.67 \text { to } 2.79)\end{array}$ & $\begin{array}{l}25.13 \pm 2.19 \\
25.54 \pm 1.89 \\
0.42(-0.29 \text { to } 1.12)\end{array}$ \\
\hline \multicolumn{4}{|l|}{ Dual-task cost (\%), mean (SD) } \\
\hline $\begin{array}{l}\text { Baseline } \\
16 \text { weeks } \\
\text { Change from baseline ( } 16 \text { weeks - baseline) }(95 \% \text { Cl) }\end{array}$ & $\begin{array}{l}38.38 \pm 23.06 \\
13.54 \pm 15.96 \\
-24.83(-34.79 \text { to }-14.87)\end{array}$ & $\begin{array}{l}39.89 \pm 28.64 \\
28.46 \pm 19.13 \\
-11.43(-27.40 \text { to } 4.54)\end{array}$ & $\begin{array}{l}38.58 \pm 22.19 \\
47.69 \pm 34.53 \\
9.11(-2.69 \text { to } 20.92)\end{array}$ \\
\hline \multicolumn{4}{|l|}{ Trail Making Test B (seconds), mean (SD) } \\
\hline Baseline & $95.36 \pm 8.08$ & $95.38 \pm 11.68$ & $95.49 \pm 11.35$ \\
\hline 16 weeks & $69.81 \pm 13.91$ & $84.67 \pm 14.67$ & $93.39 \pm 11.59$ \\
\hline Change from baseline (16 weeks - baseline) (95\% Cl) & $-25.55(-32.94$ to -18.17$)$ & $-10.70(-14.70$ to -6.69$)$ & $-2.11(-6.74$ to 2.53$)$ \\
\hline \multicolumn{4}{|l|}{ Forward Digit Span (points), mean (SD) } \\
\hline Baseline & $10.57 \pm 1.41$ & $10.64 \pm 1.05$ & $10.63 \pm 1.25$ \\
\hline 16 weeks & $12.48 \pm 1.73$ & $12.32 \pm 1.78$ & $11.25 \pm 2.05$ \\
\hline Change from baseline (16 weeks - bassline) (95\% Cl) & $1.91(1.26$ to 2.56$)$ & $1.62(1.08$ to 2.28$)$ & $0.63(-0.02$ to 1.27$)$ \\
\hline \multicolumn{4}{|l|}{ Backward Digit Span (points), mean (SD) } \\
\hline Baseline & $8.26 \pm 0.86$ & $8.27 \pm 1.42$ & $8.33 \pm 1.13$ \\
\hline 16 weeks & $9.87 \pm 1.25$ & $9.32 \pm 1.56$ & $8.13 \pm 1.98$ \\
\hline Change from baseline (16 weeks - bassline) $(95 \%$ Cl) & 1.61 (1.09 to 2.13$)$ & 1.05 (0.51 to 1.58$)$ & $-0.21(-1.11$ to 0.69$)$ \\
\hline \multicolumn{4}{|l|}{ Verbal Fluency (number of animals named), mean (SD) } \\
\hline Baseline & $13.26 \pm 1.66$ & $13.36 \pm 0.95$ & $13.71 \pm 1.39$ \\
\hline 16 weeks & $17.22 \pm 2.59$ & $15.59 \pm 2.30$ & $14.13 \pm 1.36$ \\
\hline Change from baseline (16 weeks - bassline) $(95 \% \mathrm{Cl})$ & $3.96(4.95$ to 2.97$)$ & 2.23 (1.09 to 3.36$)$ & $0.42(-0.24$ to 1.08$)$ \\
\hline
\end{tabular}

SD Standard deviation

\section{Test-Retest Reliability}

Reliability estimates for the cognitive measures are presented in Table 4. ICCs from the 2-week test-retest analyses were quite high, ranging from 0.87 (MoCA) to 0.92 (Verbal Fluency).

\section{Discussion}

In this 3-arm feasibility trial, we examined the feasibility, acceptability, and safety of implementing a newly developed cognitively enhanced online Tai Ji Quan intervention, along with a standard Tai Ji Quan training and stretching exercise, for older adults with MCI. Feasibility was demonstrated by the successful online program implementation with acceptable fidelity, intervention 
compliance, and study retention. The newly developed intervention was perceived to be acceptable and satisfactory by the participants. Overall, participants in the study completed the assigned exercise intervention, with no major intervention-related serious or moderate events observed across all intervention groups.

Recruitment of the study population was shown to be feasible for such an online (virtual) intervention. We were able to successfully carry out our online recruitment procedures (prescreening per the study eligibility criteria, study consent) with an approximately 2-to-1 screening-to-enrollment ratio, or a recruitment rate of $55 \%$. Of the different recruitment promotion methods used, mass mailing and word of mouth were the most successful methods of reaching out to the target population [27]. The recruitment started in the state of Oregon and extended to other states, reflecting general interest in both the use of and receiving telehealth services [41, 42]. Even with different time zones, our online class was able to accommodate older adults in other states, depending on individual schedules. This was encouraging because it potentially helps the program reach a broader population, including those who may have been left behind [43] and those who are underserved and living in remote areas or rural settings. The intervention attrition (dropout) rate was $11 \%$, and we were able to retain $94 \%$ of the study participants. Reasons for dropping out of the study were similar to previous in-person interventions $[27,28$, $31,32]$, and none of the reasons were related to the online class set-up or use of Zoom technology.

To accommodate online data collection, modifications were made in some cognitive measures, which were developed based on in-person protocols. Even with alterations in measurement set-up and testing protocols, we found no safety or technical issues during online assessment, which was conducted via videoconferencing at the participants' homes, thus showing the feasibility of collecting cognitive and mobility outcome data remotely and with good test-retest reliability. These results, along with those we demonstrated previously [27], provide support for ascertaining online data when, under extenuating circumstances, in-person data collection approaches are not feasible, such as with COVID-19 or for those living in remote areas. Finally, changes (in the sense of improvement) from baseline in the measured cognitive and dual-task outcomes were observed consistently for the cognitively enhanced group whereas the standard Tai Ji Quan training group improved in all cognitive measures but dual-task cost. No change in the outcome measures was observed for the stretching exercise group.

While the cognitive health benefits of Tai Ji Quan for cognitively intact and impaired older adults have been documented [16-18], to our knowledge this is one of the few studies that have considered Tai Ji Quan training with explicit integration of cognitive-motor dual-task performance and practice. Previously, we have shown the feasibility of an augmented dual-task Tai Ji Quan intervention for balance training and falls prevention for older adults with MCI [27]. In this feasibility study, we enhanced, and therefore extended, our prior work by synergistically combining the physical and cognitive elements underlying the goal-directed Tai Ji Quan movements to boost cognition in older adults with MCI. Our approach, which aligns with contemporary training strategies [44-46], extends current Tai Ji Quan training approaches by involving concurrent, interactive physicalcognitive exercises aimed at stimulating and challenging multiple cognitive domains. Unique features of our new intervention include (a) Tai Ji Quan practice intertwined with cognitive exercises, (b) active engagement of and interaction with participants by the instructor during online class sessions, and (c) a manual-driven protocol for online instructional teaching.

\section{Strengths and Limitations}

A notable strength of this study is the rigorous 3-arm design and methodologies that involve both assessing trial process feasibility and exploring trial outcome measures. Other strengths include successful recruitment of the target population, implementation of online exercise intervention classes, and satisfactory intervention compliance and retention rates.

The feasibility study also has some notable limitations. First, no sample size calculations were performed due to the pilot nature of this study. Therefore, the results on the cognitive and dual-task outcomes are preliminary and serve as a trend in outcome measures for future trials. Similarly, our study population can only be confined to the eligibility criteria applied in this study. Second, our study design does not include a cognitive training arm. Therefore, we may not know whether multitasking cognitive training alone or Tai Ji Quan with multitasking training works equally in terms of potential efficacy of our reported cognitive outcomes. However, the inclusion of the standard Tai Ji Quan training in the study helps discriminate task-specificity in cognition and control for placebo effects, and thus allows a rigorous evaluation of the incremental value of adding a cognitive training component to our enhanced Tai Ji Quan training approach. Third, due to limitations associated with our online recruitment and assessment protocols, we were unable to include additional MCI screening measures, such as executive function, attention, language, or visuospatial skills, which would be more ideal in capturing the outcomes for the target population [47]. The same limitation exists in our cognitive outcome measures, which are to 
be used in our future efficacy trial. Inclusion of additional domain-specific outcome measures such as visuospatial, cognitive inhabitation, and memory-related functions would provide a better representation of cognition that may have been benefited from our intervention.

\section{Conclusions}

In this 3-arm intervention feasibility trial, we demonstrated the feasibility, acceptability, and safety of a newly developed cognitively enhanced Tai Ji Quan intervention, delivered to home settings via online videoconferencing, for community-dwelling older adults with MCI. Additional feasibility data included collecting outcome data remotely and trends in cognitive and dual-task outcomes resulting from a 16-week intervention. We conclude that the outcomes from this feasibility study form the basis for a full-scale randomized online intervention trial that examines the effectiveness of our cognitively enhanced online Tai Ji Quan intervention for older adults with MCI.

\section{Abbreviations}

CDR: Clinical Dementia Rating; COVID-19: Coronavirus disease 2019; ICC: Intraclass correlation coefficient; MCl: Mild cognitive impairment; MMSE: MiniMental State Evaluation; MoCA: Montreal Cognitive Assessment; TUG: Timed Up and Go; TMT-B: Trail Making Test B.

\section{Acknowledgements}

Not applicable.

\section{Authors' contributions}

Study conception and design, FL; literature search, FL, PH, KF, KW; intervention design and training, FL, PH; trial recruitment, operationalization, data collection, statistical analysis, paper drafting and interpretation, FL, PH. All authors contributed to editing and approved the final text.

\section{Funding}

The study reported in this manuscript was supported by the National Institute on Aging (3R01AG059546-02S1). The opinions expressed are those of the authors and do not necessarily represent the official views of the National Institute on Aging.

\section{Availability of data and materials}

The de-identified data that support the findings of this study are available from the corresponding author upon reasonable request. Restrictions may apply to preserve participant confidentiality.

\section{Declarations}

Ethics approval and consent to participate

Ethical approval was provided the Institutional Review Board (IRB) of the Oregon Research Institute (Approval no. FWA00005934). All participants gave informed consent to take part in the research. All methods were carried out in accordance with our IRB-approved protocol.

\section{Consent for publication}

Not applicable.

\section{Competing interests}

The authors declare that they have no competing interests.

\section{Author details}

${ }^{1}$ Oregon Research Institute, Eugene, Oregon, OR 97403, USA. ²Department of Exercise and Health Science, Willamette University, Salem, OR 97301, USA. ${ }^{3}$ McKenzie Willamette Medical Center, Springfield, OR 97477, USA. ${ }^{4}$ Knight Cancer Institute, Oregon Health \& Science University, Portland, OR 97239, USA.

Received: 16 November 2021 Accepted: 29 December 2021 Published online: 25 January 2022

References

1. Harada CN, Natelson Love MC, Triebel KL. Normal cognitive aging. Clin Geriatr Med. 2013;29(4):737-52.

2. National institute on Aging. Memory, forgetfulness, and aging: What's normal and what's not? Available at: https://www.nia.nih.gov/health/ memory-forgetfulness-and-aging-whats-normal-and-whats-not. Accessed 16 Nov 2021.

3. Petersen RC, Smith GE, Waring SC, Ivnik RJ, Tangalos EG, Kokmen E. Mild cognitive impairment: clinical characterization and outcome. Arch Neurol. 1999;56(3):303-8.

4. Campbell NL, Unverzagt F, LaMantia MA, Khan BA, Boustani MA. Risk factors for the progression of mild cognitive impairment to dementia. Clin Geriatr Med. 2013;29(4):873-93.

5. Prince MJ, Wimo A, Guerchet MM, Ali GC, Wu YT, et al. World Alzheimer Report 2015-The Global Impact of Dementia: An analysis of prevalence, incidence, cost and trends. Available: https://www.alz.co.uk/research/ world-report-2015. Accessed 11 Sept, 2021.

6. GBD. 2016 Dementia Collaborators. Global, regional, and national burden of Alzheimer's disease and other dementias, 1990-2016: a systematic analysis for the Global Burden of Disease Study 2016. Lancet Neurol. 2019;18(1):88-106.

7. Livingston G, Huntley J, Sommerlad A, Ames D, Ballard C, Banerjee S, et al. Dementia prevention, intervention, and care: 2020 report of the Lancet Commission. Lancet. 2020;396:413-46.

8. Voss MW, Nagamatsu LS, Liu-Ambrose T, Kramer AF. Exercise, brain, and cognition across the life span. J Appl Physiol. 2011;111:1505-13.

9. Gregory M, Gill D, Petrella R. Brain health and exercise in older adults. Curr Sports Med Rep. 2013;12(4):256-71.

10. Biazus-Sehn LF, Schuch FB, Firth J, de Souza SF. Effects of physical exercise on cognitive function of older adults with mild cognitive impairment: A systematic review and meta-analysis. Arch Gerontol Geriatr. 2020;89:104048.

11. Wollesen B, Voelcker-Rehage C. Training effects on motor-cognitive dualtask performance in older adults. Eur Rev Aging Phys Act. 2014;11:5-24.

12. Gregory M, Gill D, Petrella R. Brain health and exercise in older adults. Curr Sports Med Rep. 2013;12(4):256-71.

13. Kramer AF, Larish JF, Strayer DL. Training for attentional control in dual task settings: a comparison of young and old adults. J Exp Psychol. 1995:1:50-76.

14. Li F. Transforming traditional tai ji quan techniques into integrative movement therapy_-Tai Ji Quan: moving for better balance. J Sport Health Sci. 2014;3(1):9-15.

15. Li F. The public health benefits of Tai Ji Quan — Addressing the unmet needs of aging populations in the 21st century. J Sport Health Sci. 2016;5:304-7

16. Lin $R$, Cui S, Yang J, Yang H, Feng Z, Dietlind LD, et al. Effects of Tai Chi on patients with mild cognitive impairment: A systematic review and meta-analysis of randomized controlled trials. BioMed Res Int. 2021;2021(12):5530149.

17. Gu R, Gao Y, Zhang C, Liu X, Sun Z. Effect of Tai Chi on Cognitive Function among Older Adults with Cognitive Impairment: A systematic review and meta-analysis. Evid Based Complement Alternat Med. 2021;2021:6679153.

18. Wayne PM, Walsh JN, Taylor-Piliae RE, et al. Effect of tai chi on cognitive performance in older adults: systematic review and meta-analysis. J Am Geriar Soc. 2014;62:25-39.

19. Bishnoi A, Hernandez ME. Dual task walking costs in older adults with mild cognitive impairment: a systematic review and meta-analysis. Aging Mental Health. 2021;25(9):1618-29. 
20. Yang Q, Tian C, Tseng B, Zhang B, Huang S, Jin S, et al. Gait change in dual task as a behavioral marker to detect mild cognitive impairment in elderly persons: A systematic review and meta-analysis. Arch Phys Med Rehabil. 2020;101(10):1813-21.

21. NORC at the University of Chicago Needs Assessment and Environmental Scan Report: Maintaining Physical and Mental Well-Being of Older Adults and Their Caregivers During Public Health Emergencies. Bethesda, MD: NORC at the University of Chicago; 2021. Available at: https://www.norc. org/PDFs/Maintaining\%20Physical\%20and\%20Mental\%20Well/ESand NAReportNarrative.pdf. Accessed 12 Nov 2021.

22. Eldridge SM, Chan CL, Campbell MJ, Bond CM, Hopewell S, Thabane $L$, et al. CONSORT 2010 statement: extension to randomised pilot and feasibility trials. Pilot Feasibility Studu. 2016;2:64.

23. Petersen RC. Mild cognitive impairment as a diagnostic entity. J Int Med. 2004;256:183-94.

24. Morris JC. The Clinical Dementia Rating (CDR): current version and scoring rules. Neurol. 1993;43:2412-4.

25. Folstein MF, Folstein SE, McHugh PR. "Mini-mental state": a practical method for grading the cognitive state of patients for the clinician. J Psychiatr Res. 1975;12(3):189-98.

26. Sheikh JI, Yesavage JA. Geriatric Depression Scale (GDS): Recent evidence and development of a shorter version. Clin Gerontologist. 1986;5(1-2):165-73.

27. Li F, Harmer P, Voit J, Chou LS. Implementing an online virtual falls prevention intervention during a public health pandemic for older adults with mild cognitive impairment: A feasibility trial. Clin Interv Aging. 2021;16:973-83.

28. Li F, Harmer P, Fitzgerald K, Eckstrom E, Akers L, Chou LS, et al. Effectiveness of a therapeutic Tai Ji Quan intervention vs multimodal exercise intervention to prevent falls among older adults at high risk of falling: a randomized clinical trial. JAMA Intern Med. 2018;178(10):1301-10.

29. Li F, Harmer P, Liu Y, Chou LS, Tai J. Quan and global cognitive function in older adults with cognitive impermanent: a pilot study. Arch Gerontol Geriatr. 2014;58(3):434-9.

30. American College of Sport Medicine. ACSM's Guidelines for Exercise Testing and Prescription. 10th ed. Philadelphia: Lippincott Williams \& Wilkins; 2010.

31. Li F, Harmer P, Fitzgerald K. Implementing an evidence-based fall prevention intervention in community senior centers. Am J Public Health. 2016;106(11):2026-31.

32. Li F, Harmer P, Fisher KJ, McAuley E, Chaumeton N, Eckstrom E, et al. Tai Chi and fall reductions in older adults: a randomized controlled trial. J Gerontol A Biol Sci Med Sci. 2005;60(2):187-94.

33. Bernstein $I H$, Lacritz $L$, Barlow $C E$, Weiner MF, DeFina LF. Psychometric evaluation of the Montreal Cognitive Assessment (MoCA) in three diverse samples. Clin Neuropsychol. 2011;25(1):119-26.

34. Podsiadlo D, Richardson S. The timed "Up \& Go": a test of basic functional mobility for frail elderly persons. J Am Geriatr Soc. 1991;39(2):142-8.

35. Li F, Harmer P, Chou LS. Dual-task walking capacity mediates Tai Ji Quan impact on physical and cognitive function. Med Sci Sports Exerc. 2019;51(11):2318-24

36. Corrigan JD, Hinkeldey MS. Relationships between Parts A and B of the Trail Making Test. J Clin Psychol. 1987;43:402-9.

37. Sager MA, Hermann BP, LaRue A, Woodard JL. Screening for dementia in community-based memory clinics. Wisconsin Med J. 2006;105(7):25-9.

38. Wechsler D. Wechsler Adult Intelligence Scale - IV. Pearson: Bloomington. MN: Psychological Corporation; 1980.

39. Craig CL, Marshall AL, Sjöström M, Bauman AE, Booth ML, Ainsworth BE, et al. International physical activity questionnaire: 12-country reliability and validity. Med Sci Sports Exerc. 2003;35(8):1381-95.

40. Leon AC, Davis LL, Kraemer HC. The role and interpretation of pilot studies in clinical research. J Psychiatr Res. 2011;45(5):626-9.

41. Choi NG, Dinitto DM, Marti CN, Choi BY. Telehealth use among older adults during COVID-19: Associations with sociodemographic and health characteristics, technology device ownership, and technology learning. J Appl Gerontol. 2021;5:7334648211047347.

42. Ng BP, Park C. Accessibility of telehealth services during the COVID-19 pandemic: A cross-sectional survey of Medicare Beneficiaries. Prev Chronic Dis. 2021;18:210056.
43. Lam K, Lu AD, Shi Y, Covinsky KE. Assessing telemedicine uneasiness among older adults in the United States during the COVID-19 pandemic. JAMA Intern Med. 2020;180(10):1389-91.

44. Lauenroth A, Loannidis AE, Teichmann B. Influence of combined physical and cognitive training on cognition: a systematic review. BMC Geriatr. 2016;16:141.

45. Gavelin HM, Dong C, Minkov R, Bahar-Fuchs A, Ellis KA, Lautenschlager NT, et al. Combined physical and cognitive training for older adults with and without cognitive impairment: A systematic review and network meta-analysis of randomized controlled trials. Ageing Res Rev. 2021;66:101232.

46. Yang C, Moore A, Mpofu E, Dorstyn D, Li Q, Yin C. Effectiveness of combined cognitive and physical interventions to enhance functioning in older adults with mild cognitive impairment: a systematic review of randomized controlled trials. Gerontologist. 2019;23;60(8):633-42.

47. Albert MS, DeKosky ST, Dickson D, Dubois B, Feldman HH, Fox NC, et al. The diagnosis of mild cognitive impairment due to Alzheimer's disease: Recommendations from the National Institute of Aging-Alzheimer's Association workgroups on diagnostic guidelines for Alzheimer's disease. Alzheimers Demen. 2011;7(3):270-9.

\section{Publisher's Note}

Springer Nature remains neutral with regard to jurisdictional claims in published maps and institutional affiliations.

Ready to submit your research? Choose BMC and benefit from:

- fast, convenient online submission

- thorough peer review by experienced researchers in your field

- rapid publication on acceptance

- support for research data, including large and complex data types

- gold Open Access which fosters wider collaboration and increased citations

- maximum visibility for your research: over $100 \mathrm{M}$ website views per year

At BMC, research is always in progress.

Learn more biomedcentral.com/submissions 